\title{
EARTHWORK VOLUMETRICS WITH UNMANNED AERIAL VEHICLES: A COMPARATIVE STUDY
}

\author{
Raid Al-Tahir* and Travis Barran \\ Department of Geomatics Engineering and Land Management, \\ The University of the West Indies, Trinidad and Tobago \\ ${ }^{1}$ Email: raid.altahir@sta.uwi.edu*(Corresponding author)
}

\begin{abstract}
The recent evolution of Unmanned Aerial Vehicles (UAVs) as measuring instruments has become attractive for many surveying applications in civil engineering including the volumetric computations of earthworks in the construction of a highway. The application of softcopy photogrammetry to UAV acquired imagery has the potential to reduce data acquisition costs, time and with suitable accuracy for earthworks mapping and volumetrics. Their performance, however, is not well understood for these applications. This investigation tests the ability of the UAVs and photogrammetric software to generate volumes for the layers of material used in road construction, as well as to analyse their accuracies and limitations. Specifically, this study assesses the feasibility of UAV-based surveying in generating volumes for general earthworks in highway/road construction. Additionally, the study compares the performance of UAV-based surveying to that of Total Station surveying. Performance is evaluated along the required time for image acquisition and generating the final products, the required personnel and overall cost of survey. A segment of the Churchill Roosevelt Extension to Manzanilla was used in the assessment.
\end{abstract}

Keywords: Photogrammetry, Unmanned Aerial Vehicles, Earthwork Computation, Highway Construction.

https://doi.org/10.47412/KLNQ8966

\section{Introduction}

\subsection{Background}

Attaining volumetric data for earthworks are important tasks in many engineering disciplines such as highway/road construction and the mining industry. For highway construction, the physical works on the site include general earthwork activity such as: clearing and grubbing, excavation, hauling and placing of soil, gravel or other material found on the surface of the earth. The measurement of all such materials must be carried out before and after the scheduled activity. 
Currently, the methods used in acquiring data for volume calculations consists of the employment of a survey team to perform on site topographic measurements to provide required quantities for the relevant pricing of an activity. The general methodology of measurement involves performing the surveying technique before and after the completion of the earthwork activity, which would be used to create two surfaces. These two surfaces, when subtracted would give the volume of material excavated or filled. The volume measurements are traditionally executed using surveying techniques such as Total Station surveys, Global positioning system (RTK) (Hugenholtz, Walker, Brown and Myshak 2015). These methods have proven to be labour intensive and fairly time consuming.

The constant advancements in Unmanned Aerial Vehicle (UAV) systems and automated photogrammetric software have made attaining geospatial data more cost effective and less time consuming. The recent evolution of UAVs as measuring instruments has become attractive for many surveying applications in civil engineering including the volumetric computations of earthworks in the construction of a highway. Their performance, however, is not well understood for these applications. More research is needed to test the ability of the UAV and photogrammetric software to generate volumes for the layers of material used to build the road structure and to analyse the accuracies and limitations found.

The purpose of this investigation is to analyse the performance of UAV systems in acquiring 3D mapping data for large earthmoving construction sites. This study aims to compare and assess the feasibility of UAV surveying in generating volumes for general earthworks in highway/road construction to that of Total Station surveying.

\subsection{Unmanned Aerial Vehicles (UAVs)}

The Unmanned Aerial Vehicle is an aircraft designed to operate with no human pilot on board. UAVs provide an unprecedented solution to the problem of capturing fine, scale-specific, high spatial resolution imagery, for a variety of remote sensing applications. They provide the flexibility for an operating team to collect data under conditions not typically conventional for traditional, aircraft based, aerial photo acquisition, as well as the ability to fly at a lower altitude (Remondino, Barazzetti, Nex, Scaioni, and Sarazzi, 2012).

With the recent evolution of technology, highly accurate global navigation and positioning systems (GNSS) were integrated with the UAV at a low cost, allowing the aircraft's position to be determined within a global reference system, in real time. The development of light cost-efficient gyroscopes and digital cameras further enhanced the performance, payload, endurance and flexibility for diverse and reliable applications of UAV systems. In parallel to these hardware advancements, computer algorithms have been developed to produce high-quality photogrammetric products from UAV images (Fernandes et al. 2015). It is through these advancements that automated photogrammetric software can be used to reconstruct a scene in 3D. 


\subsection{Viability of UAV and Photogrammetry in Volume Calculations}

One of the most important components in highway/roadway engineering is the reliable and accurate calculation of earthwork volumes. These volume calculations can influence the selection of the highway's alignment (route), its construction process and hence its building cost. The use of UAVs in construction applications are quickly becoming a cost-effective alternative to traditional approaches, particularly for small- to medium sized earthwork projects. Only a few studies have been conducted to assess their error performance in a realistic field study. One study concluded that UAV-based volumetrics can yield measurements similar to conventional techniques and can offer a more cost effective, efficient and safer technique for surveying earthwork projects (Hugenholtz, Walker, Brown and Myshak 2015).

Harwin and Lucieer (2012) examined the accuracy of georeferenced point clouds created using UAV imagery and compared to a Total Station survey. The study area consisted of a 100-meter section of shelter coast in southern Tasmania, Australia. The tests results showed a 2.5-4 cm difference in the points generated from the UAV images and the points from the Total Station. The authors concluded that the method of using UAV images to generate 3D products is capable of monitoring sub-decimetre terrain changes in applications such as erosion in costal environments (Harwin and Lucieer, 2012).

In another study, the point cloud generated from UAV images was tested against points measured using tachymetry over a large parking lot. Using nearly 500 measured control points, the test yielded an average positional error of $5.6 \mathrm{~cm}$ and a height error of $2.5 \mathrm{~cm}$ (Neitzel and Klonowski 2011). In a follow-up study, the same test bed was imaged and compared against the results from a Total Station survey. The modified features of this experiment included: integrated georeferencing, advanced export functions, script support, optimizing camera parameters and point clouds with the help of measured reference points. The maximum positional error was reduced to $4.9 \mathrm{~cm}$ and an average height error of $-1.1 \mathrm{~cm}$. The differences between the volumes generated by the two methods for three test piles ranged from 8-16\% (Siebert and Teizer, 2014).

The same study indicated that the UAV-based mapping produced more than 2 million points (with a density of 561 points $\left./ \mathrm{m}^{2}\right)$ in comparison to only 350 points $\left(0.02\right.$ points $\left./ \mathrm{m}^{2}\right)$. Additionally, there was a significant reduction on the overall time needed for the mapping task from $660 \mathrm{~min}$ for the Total Station survey to only $135 \mathrm{~min}$ for the UAV-based mapping. While the vertical accuracy for the targets was almost the same for both techniques, the horizontal accuracy was three times lower for the UAV-based mapping (Siebert and Teizer, 2014).

\section{Data Acquisition and Processing}

The selected site for the data acquisition was the Churchill Roosevelt Highway Extension to Manzanilla (CRHEM), located to the east of the Cumoto road and passing through the Forest reserve. The specific study area is a segment of the proposed road, along the west bound lane of the highway from chainages $7+380$ to $7+460$ ( 80 meters). The width of the road section is 17.45 meters starting from the centre of median to the end of the proposed shoulder. The specifications 
require a thickness of $600 \mathrm{~mm}$ from the top of the subgrade to the top of the subbase. The subbase material is to be placed on top of the finished subgrade and compacted to $98 \%$ compaction.

The following sections discusses the steps associated with preparing, capturing and processing photogrammetric data obtained from the UAV survey that include flight planning, GCP measurements, image acquisition by the UAV, orientation and image processing for 3D extraction.

\subsection{Reconnaissance and Flight Planning}

A reconnaissance exercise was made when a specific small area (80m x $17.45 \mathrm{~m})$ was chosen to perform the volumetric analysis. The minimum flying height that could be maintained throughout the UAV's flight, was determined based on the height of the trees and surrounding features. Accordingly, it was determined that the minimum flying height would be 55 meters which gave a Ground Sample Distance of $1.58 \mathrm{~cm} /$ pixel. The percentage of front and side overlap appropriate for stereoscopic measurement was determined $80 \%$ and $65 \%$ respectively. The percentage of front and side overlap was kept constant throughout the two missions. DroneDeploy Mapping software was then used on an android device to set the parameters for the flight plan such as flying altitude, percentage of overlap, grid size and flying speed.

\subsection{Establishing Ground Control Points (GCPs)}

Two types of photo identifiable GCPs were used in this project. The first was a wooden peg drove flush into the surface and two lines were spray painted across it in the form on an ' $X$ '. The second type of GCP, which was used in the second flight mission, was a cardboard square which measured $13 \times 13$ inches. A circle of 6-inch diameter was spray painted to a sheet of paper and then glued to the cardboard square.

Given the flat terrain and the small area of interest, nine GCPs for the first flight were placed evenly throughout the road segment in a grid pattern. The second mission involved the collection of 6 GCPs only considering that the area of interest (AOI) was refined to a smaller area. The GCPs were measured using a Leica TCR 305 Total Station and were coordinated based on the existing Geodetic Network in the site.

\subsection{Acquisition of UAV Imagery}

The small lightweight quadcopter DJI Mavic Pro was used to acquire aerial imagery over the study area. The DJI Mavic Pro was equipped with a FC220 camera that had a focal length of $4.73 \mathrm{~mm}$ and a resolution of $4000 \times 3000$ pixels. It uses GPS and GLONASS systems to geolocate itself to a meter precision.

The relevant flight parameters entered in the DroneDeploy's user interface on a smart phone. For the first flight, the flying height was set at 55.2 meters with a coverage area of $14900 \mathrm{~m}^{2}$ which was larger than the actual AOI area. The front and side overlaps were set as determined in the planning stage. The drone collected 159 vertical images in about 10 minutes while flying at a speed of $11 \mathrm{~km} / \mathrm{hr}$. 
For the second flight mission, the flying height was set at 45 meters with a coverage area of $4046 \mathrm{~m}^{2}$. The flight coverage was reduced so imagery was taken specifically to the AOI. This resulted in a smaller number of images and hence a shorter processing time. The front and side overlap were maintained at $80 \%$ and $65 \%$ respectively and the camera faced vertically downward during the data collection. 34 images were collected in the second flight of 3 minutes flight time while the flight speed was maintained at $11 \mathrm{~km} / \mathrm{hr}$.

\subsection{Processing of UAV Imagery}

Agisoft Photoscan Professional version 1.3 was used to process the images and output the required surface data. It is a stand-alone software that performs photogrammetric processing of digital images and generates 3D spatial data. The aerial photos were added into Agisoft as a group (Fig. 1). For the processing of the subgrade, 54 images were imported whilst 27 images were imported for the processing of the subbase. Agisoft then searched each individual photo for common features among images to be used as tie points and preliminary orientation. The Align tool was used to complete this process, which also created a sparse point cloud that can be used for initial generation of elevation data.

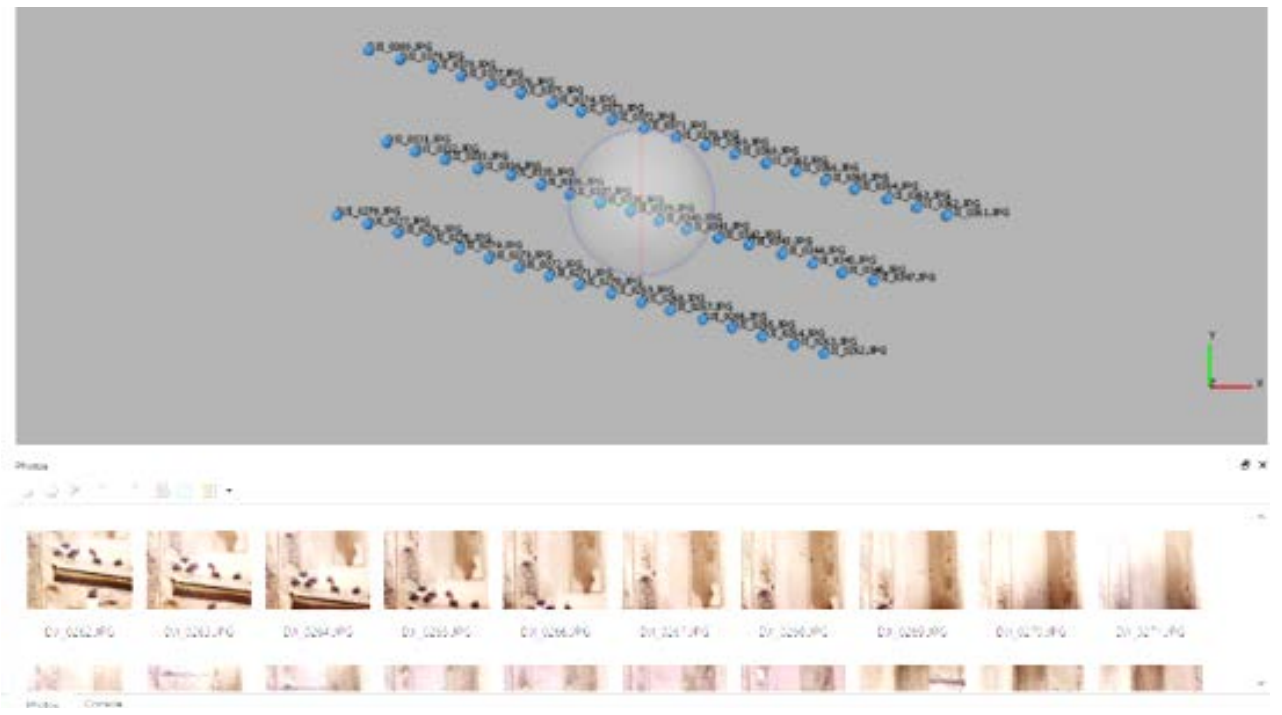

Figure 1: Images added to a chunk in Agisoft Photoscan

In order to increase the accuracy of the model, the GCPs were added to each photo by placing markers on their respective photo identifiable points, the model was then realigned by using the 'Optimize Cameras' Tool. This updated the image orientation (georeferencing) parameters and improved their accuracy.

Next, a dense point cloud was generated. The quality was set to high and the filtering was set to aggressive because the surface was relatively flat with no sudden changes or irregular features. The generated dense point cloud for the subgrade contained 3,889,801 points whilst the dense point cloud for the subbase contained 19,772,696. 


\subsection{Generating Digital Elevation Models}

A Digital Elevation Model (DEM) is a 3D representation of the bare-earth elevation model and only contains the spatial characteristics of the earth's topography. It is created by interpolating between the various coordinated mass points typically acquired by lidar or photogrammetric techniques. The Digital Elevation Model (Fig. 2) was created at this step from the dense point cloud and set to the WGS 1984 UTM zone 20 reference frame. The DEM's resolution for the subgrade was $1.47 \mathrm{~cm} /$ pixel while the DEM for the subbase had a $3 \mathrm{~cm} /$ pixel resolution.

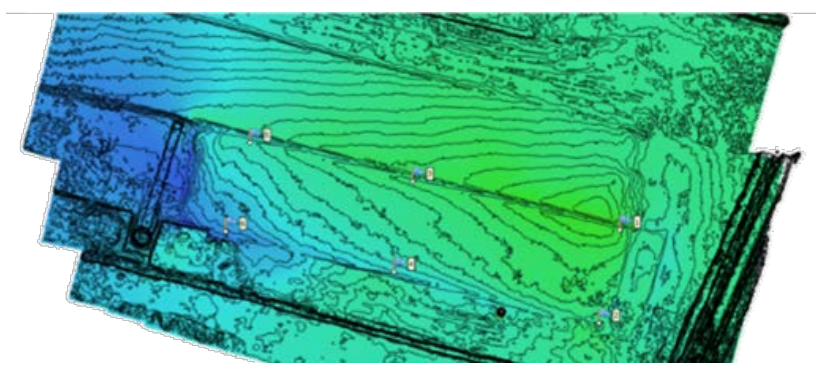

Figure 2: DEM generated from the dense point cloud

\subsection{Data Processing and Calculations}

\subsubsection{Volume Computation from Total Station Measurements}

The topographic data for both subgrade and subbase layers was extracted and saved using Leica Survey Office. The next step was to create a surface for each road layer using AutoCAD Civil 3D software. Using a total of 52 spot heights that were acquired for each layers, two separate surfaces (in the form of Triangulated Irregular Network-TIN) were created (Fig. 3). The volume of the fill surface was then calculated to be $680.29 \mathrm{~m}^{3}$ in a $1350.67 \mathrm{~m}^{2}$ area. Because no information was available on the level of compaction of the subbase layer at that time, the compaction factor was left at $100 \%$.

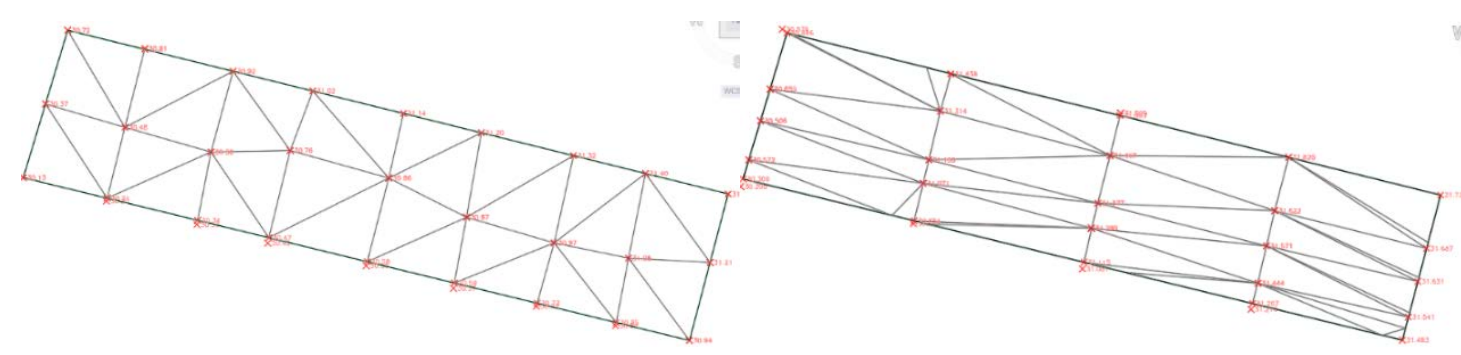

Figure 3: Created TINs for the subgrade layer surface (left), and the subbase layer (right)

\subsubsection{Volume Computation from UAV Images}

Agisoft Photoscan was used to generate a dense point cloud and a DEM for the road surface, before and after the placement of subbase material. The dense point cloud was exported as a text file that was then loaded into Autodesk RECAP. RECAP was used to crop the dense point cloud to the site 
area, perform filtering (thinning) on the point clouds, and save the output in a format suitable for Civil 3D. The points were filtered down to 0.15 meters/point (reducing the original set of 236,728 points into 118,364 points). This alone shows that data from the UAV surfaces was of a much higher resolution than the Total Station.

The final set of the filtered point for each layer were imported into AutoCAD Civil 3D and two TIN surfaces were created at a resolution of $3 \mathrm{~cm} /$ pixel (Fig. 4). The volume between the two surfaces was calculated using a Volume Calculation in the Analysis Tab. The fill factor, indicating compaction or shrinkage of the material, was left at $100 \%$. The volume of the fill surface was $743.48 \mathrm{~m}^{3}$ for the same $1350.67 \mathrm{~m}^{2}$ area.

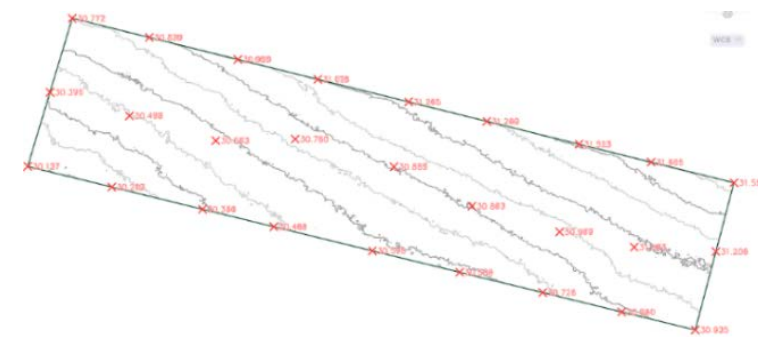

Figure 4: A UAV generated surface on AutoCAD Civil 3D in a point to surface comparison

\section{Results and Analysis}

\subsection{Assessing the Overall Accuracy of the UAV Models}

The accuracy of the 3D models generated by the UAV was assessed by the RMSE (root mean square error) values that were reported by Agisoft Photoscan. The report indicated a total RMS error of 0.018 meters in the horizontal component and 0.008-meter RSME in the vertical component (elevation) for the first flight (subgrade). The second flight yielded RMS errors of 0.03 meters in the horizontal component and 0.004 meters in the elevation. The total RMSE for both flights were $0.020 \mathrm{~m}$ and $0.031 \mathrm{~m}$ respectively. In each model, one GCP was deleted to reduce the overall RMSE of the model. The deleted GCPs contained RMS values that were significantly higher than the others and were considered as outliers.

Based on these calculations the expected discrepancy for the measurement of surface elevations should be within \pm 0.012 meters ( \pm 12 millimetres). According to the project's SMM “a tolerance of 25 millimetres above or below design elevation shall be allowed provided that the $25 \mathrm{~mm}$ above or below design elevation is not maintained for a distance longer than 15 meters". Therefore, the achieved 4- or 8-millimetre accuracy was well within the allowable range.

\subsection{Assessing the Accuracy of UAV-based Surfaces against Total Station's}

To check the accuracy of the 3D model generated by the UAV imagery, each UAV-based layer (subbase and subgrade) was checked against the topographic points collected by the Total Station. The individual surface was loaded into AutoCAD Civil 3D and the relevant Total Station points file was imported. The point to surface tool was then used to place the points on the UAV surface. These points were then exported, and the elevation data compared with that of the Total Station 
survey. The points and their height differences were plotted on an XY scatter plot to better interpret the position and the spread of the outliers.

For the subgrade layer (first flight), an average height difference between points on the UAV surface to the Total Station surface over 27 points was found to be 0.038 meters with a standard deviation of 0.05 meters from the mean. Using the value for two standard deviations from the mean $(0.12 \mathrm{~m})$, five outliers were found and deleted, bringing the average height difference down to 12 millimetres, which is within the expected elevation precision. The relevant scatter plot (Fig. 5) indicates that all the outliers were situated along a line on the northern side of the road section. This indicates that a systematic error had occurred during the data collection that can be avoided through a better planning.

For the comparison of the subbase layer, the average of the height differences of 23 points was calculated to be -0.041 meters while the standard deviation was $0.049 \mathrm{~m}$. The value for two times the standard deviation $(0.097 \mathrm{~m})$ was used to eliminate outliers from the data set which brought the value for the mean down to $0.029 \mathrm{~m}$. When one standard deviation was used, eight outliers were removed, bringing the average down to 0.016 meters; 2 millimetres over the expected precision. From the scatter plot for the subbase measurements (Fig. 6), the differences in the subbase layer was distributed randomly throughout the surface. This may be attributed to the fact that the UAV dense points would have represented the surface at a higher resolution than the Total Station, thus highlighting the irregularities in the unfinished subbase layer.

\subsection{Comparing Computed Volumes}

The computed volume using the Total Station surfaces was $680.290 \mathrm{~m}^{3}$, while the calculated volume based on the UAV data was $743.480 \mathrm{~m}^{3}$. This represents a $+62.990 \mathrm{~m}^{3}(+9.26 \%)$ difference between the two sources of data. The UAV volume was expectedly higher than the Total Station volume because of the much greater resolution of the surfaces used in the calculation. Since fewer points were taken with the Total Station, it is expected that the UAV measurement provides a more accurate earth volume estimate (Siebert, Teizer 2014). To avoid the over- and underestimating of the volume, a terrestrial laser scanner could have been utilized to produce a dense point cloud similar to the UAV, to compare the results more accurately.

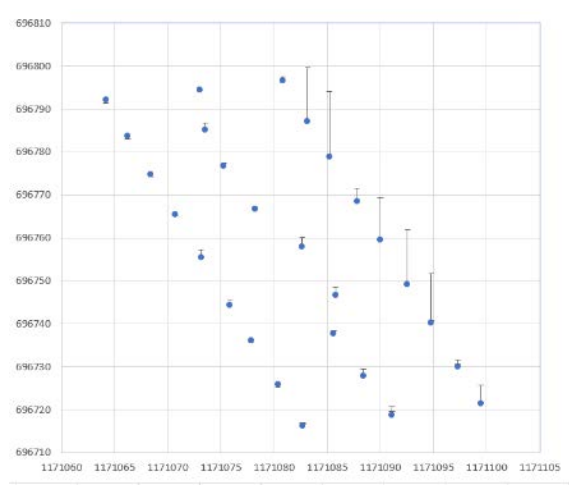

Figure 5: Scatter plot for subgrade measurements

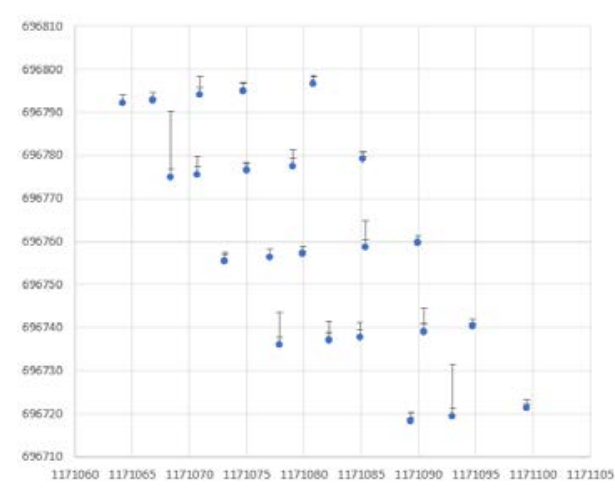

Figure 6: Scatter plot for subbase measurements 


\subsection{Comparing Required Time}

Theoretically the time taken for the UAV data acquisition should be significantly shorter than acquiring the same data using conventional surveying techniques. However, because of the small AOI, this significant difference in data acquisition time may not be realized. The results show that the UAV took 10 minutes to cover $24281 \mathrm{~m}^{2}$ (6 Acres) in the first mission. The second flight was refined to the area of interest, cutting the area coverage down to $4046 \mathrm{~m}^{2}$ (1 Acre) and the flight time to 3 minutes. The data acquisition process using the Total Station took 10 minutes to survey $1350 \mathrm{~m}^{2}$ of the road section.

The processing of the UAV imagery, however, took a considerably longer time than the post processing of the Total Station data. The UAV processing lasted approximately 24 hours while the Total Station processing lasted just 8 minutes. The lengthy processing time for the UAV images was down to a few reasons. Firstly, the first mission covered too large of an area, overshooting the area of interest by 22,931 $\mathrm{m}^{2}$. This first mission required the software to process 159 images to generate a dense point cloud for analysis. In the second mission this coverage was largely reduced, only requiring 34 images to be captured with the flight parameters remaining the same (80\% front, $65 \%$ side overlap). The processing of this image chunk was somewhat faster than the first flight.

The second reason for the extensive amount of time it took for post processing of images, was the high accuracy the filtering applications was set at in creating the 3D models. In creating the dense point cloud, which was the longest process in the image processing, the accuracy was set to high and the depth filtering set to mild. The depth filtering allows the software to smoothen edges in flat terrain imagery, or to refine the details in imagery which contain several features. Concerning this project, the depth filtering could have been set to aggressive in the first flight processing which would have maintained the accuracy of the dense point cloud, since the AOI was uniform. For the second processing, the depth filter was set to aggressive to speed up the time.

The time taken by the UAV to acquire data for the road surface was less than half the time it took using Conventional Total Station surveying. As the length of road section increases, this time difference between methods tends to increase further. It shows that the UAV allows for faster data collection over larger areas, also allowing construction work to continue during data capture.

\subsection{Comparing Required Cost and Personnel}

An important factor, with regards to any project is the cost of acquiring the relevant data to a specific accuracy. Based on information acquired by this study, the required hardware and software to collect and process measurements by a Total Station cost a total of US\$ 66,720. On the other hand, the hardware and software for UAV-based mapping cost US\$ 30,750. Accordingly, the UAV approach costs less than $50 \%$ of the cost of the Total Station approach but resulting in a greater volume of material. The average dump truck holds between 7.6 to $10.7 \mathrm{~m}^{3}$ of material. Based on the volume calculated by the UAV method, an additional 5 to 8 loads of compacted material would be included in the bill of quantities.

Another factor in the feasibility of UAVs in road construction is the number of personnel needed for data acquisition. During the data collection of this research the UAV survey only required one 
person to operate the drone. Whereas, the Total Station survey was performed by three persons; one technician and two chainmen. On larger road sections, three chainmen are used to improve the efficiency of the task.

\section{Conclusion}

This project investigated the performance of UAVs in volumetric calculation and surface measurement in comparison to the traditional surface measurements by a Total Station. The study also made an assessment regarding the feasibility of UAV surveying with respect to accuracy, time, cost and personnel. Measurements were made using Total Station and UAV techniques on the surface of subgrade and subbase layers for a road section on the Churchill Roosevelt Highway Extension to Manzanilla construction site. Using the data from the two techniques, two values were calculated for the volume of compacted subbase material. The UAV method produced a $9.26 \%$ difference in volume to that of the Total Station volume.

The surfaces created by the UAV images were also assessed against points collected by the Total Station. The subgrade surface created using the UAV images showed an average height difference of 12 millimetres when the 5 outliers were deleted. The subbase showed a 16-millimetre difference in height between methods when eight outliers were removed. This accuracy is well within the acceptable range used for surface measurement as specified by the project's SMM (Standard Method of Measurement). The accuracy by which the GCPs were measured can be increased if GPS RTK methods are employed. This would increase the accuracy of the surface measurements and hence the overall accuracy of volume computations.

The UAV approach was proven to be a more cost and time effective solution when compared to Total Station surveying in measuring the structural layers in road construction. As higher accuracies can be attained through the precise measurement of GCPs, this renders UAV surveying as a suitable option to be used in highway construction.

\section{References}

[1] Fernández-Hernandez, J., González-Aguilera, D., Rodríguez-Gonzálvez, P. \& ManceraTaboada, J. (2015). Image-Based Modelling from Unmanned Aerial Vehicle (UAV) Photogrammetry: An Effective, Low-Cost Tool for Archaeological Applications. Archaeometry, 57(1), 128-145.

[2] Harwin, S., and Lucieer, A. (2012). Assessing the Accuracy of Georeferenced Point Clouds Produced via Multi-View Stereopsis from Unmanned Aerial Vehicle (UAV) Imagery. Remote Sensing, 4(6), 1573-1599. Retrieved 49 2019, from https://doi.org/10.3390/rs4061573

[3] Hugenholtz, C. H., Walker, J., Brown, O. W., and Myshak, S. (2015). Earthwork Volumetrics with an Unmanned Aerial Vehicle and Softcopy Photogrammetry. Journal of Surveying Engineering-asce, 141(1), 06014003.

[4] Neitzel, F. and Klonowski, J. (2011). Mobile 3d Mapping With A Low-Cost UAV System. International Archives of the Photogrammetry, Remote Sensing and Spatial Information Sciences, 39-44. 
[5] Remondino, F., Barazzetti, L., Nex, F. C., Scaioni, M., and Sarazzi, D. (2012). UAV Photogrammetry for Mapping and 3D Modeling - Current Status and Future Perspectives. ISPRS - International Archives of the Photogrammetry, Remote Sensing and Spatial Information Sciences 25-31.

[6] Siebert, S., and Teizer, J. (2014). Mobile 3D Mapping For Surveying Earthwork Projects Using an Unmanned Aerial Vehicle (UAV) system. Automation in Construction, 41(41), 1-14. 